\title{
RANDOM FIXED POINTS AND RANDOM DIFFERENTIAL INCLUSIONS
}

\author{
NIKOLAOS S. PAPAGEORGIOU \\ University of California \\ 1015 Department of Mathematics \\ Davis, California 95616 \\ (Received July 28, 1987 and in revised form October 19, 1987)
}

\begin{abstract}
In this paper, first, we study random best approximations to random sets, using fixed point techniques, obtaining this way stochastic analogues of earlier deterministic results by Browder-Petryshyn, KyFan and Reich. Then we prove two fixed point theorems for random multifunctions with stochastic domain that satisfy certain tangential conditions. Finally we consider a random differential inclusion with upper semicontinuous orientor field and establish the existence of random solutions.
\end{abstract}

KEY WORDS AND PHRASES. Random fixed point, Random solutions, Random Differential Inclusion and Abstract Differential Equations.

1980 AMS CLASSIFICATION CODE. $60 \mathrm{H} 25,47 \mathrm{H1} 0$.

1. INTRODUCTION.

Random fixed point theorems are stochastic generalizations of classical fixed point theorems and are needed in the study of random equations. Their study was initiated by the Prague school of probabilists, with the works of Hans [1] and Spacek [2]. Recently the interest in these problems was revived by the survey article of Bharucha-Re1d [3]. Since then, there has been a lot of activity in this area and several interesting results have appeared.

In this paper, we will study random fixed points in connection with random approximations and will derive stochastic analogues of some results by BrowderPetryshyn [4], KyFan [5] and Reich [6]. We also extend a random fixed point theorem proved by Engl [7] and finally we prove the existence of a solution for a random differential inclusion with an upper semicontinuous orientor field, extending this way an earlier result of the author [8] (theorem 5.1).

For the corresponding deterministic theory, we refer to the recent books of Goebel-Reich [9] for fixed points (in connection with the study of the geometry of the underlying space) and of Aubin-Cellina [10] for differential inclusions. Another nice work, bringing together the two main mathematical branches considered in this note, namely fixed point theory and differential equations, is the paper of Reich [11], where an interesting approach to fixed point theory is presented, through the existence theory of abstract differential equations. 


\section{PRELIMINARIES.}

Let $(\Omega, \Sigma)$ be a measurable space and $\mathrm{X}$ a separable Banach space. Throughout this work, we will be using the following notations:

and

$$
P_{f(c)}(X)=\left\{A_{C} X: \text { nonempty, closed (convex) }\right\}
$$

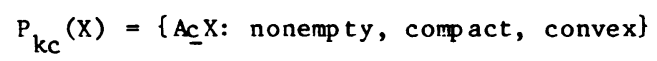

Let $\mathrm{K}: \Omega \rightarrow \mathrm{P}_{\mathrm{f}}(\mathrm{X})$ be a multifunction. We say that $\mathrm{K}\left(_{\bullet}\right)$ is measurable, if for all UcX open, we have that $K^{-}(U)=\{\omega \varepsilon \Omega: K(\omega) \cap U \neq \phi\} \varepsilon \Sigma$. It can be shown - see Himmelberg [10] - that the above definition of measurability of $K\left(_{\cdot}\right)$ is equivalent to saying that for any $z \varepsilon X$, the map $\omega+d(z, K(\omega))=\operatorname{lnf}\{\|z-x\|: x \in K(\omega)\}$ is measurable. Furthermore, if there exists a complete $\sigma$-finite measure on $\Sigma$, then the above two properties are equivalent to saying that $\operatorname{GrK}=\left\{(\omega, \mathrm{x}) \varepsilon \Omega_{\mathrm{xX}}: \mathrm{x} \varepsilon \mathrm{K}(\omega)\right\} \varepsilon \Sigma \mathrm{xB}(\mathrm{X})$, where $B(X)$ is the Borel o-field of $X$. Following Schal [12] and Engl [7], we will say that $K: \Omega 2 \rightarrow P_{f}(X)$ is separable, if it is measurable and there exists a countable set DcX s.t. $\quad c 1(D \cap K(\omega))=K(\omega)$. It is not difficult to show that if $K($.$) is measurable$ with nonempty, closed values and $K(\omega)=c l($ int $K(\omega))$ for all $\omega \varepsilon \Omega$, then $K(\cdot)$ is a separable multifunction. This is the case for example, when $K_{(}$) has closed, convex, solid values.

Let $Y, Z$ be two Hausdorff topological spaces and let $G: Y \rightarrow 2^{Z} \backslash\{\}$ be a multifunction. We say that $G_{(\bullet)}$ is upper semicontinuous (u.s.c.), if for all Uch open, $G^{+}(U)=\{y \in Y: G(y) \underline{c} U\}$ is open in $Y$. Also by $h(\ldots,$.$) we will denote the$ Hausdorff metric on $P_{f}(X)$. Recall that $\left(P_{f}(X), h\right)$ is a complete metric space.

Let $\mathrm{K}: \Omega \rightarrow \mathrm{P}_{\mathrm{f}}(\mathrm{X})$ and let $\mathrm{F}: \mathrm{GrK}+\mathrm{P}_{\mathrm{f}}(\mathrm{X})$. We say that $\mathrm{F}(.,$.$) is a random$ multifunction with stochastic domain $K(\cdot)$, if $K(\cdot)$ is measurable and for all $x \in X$ and UcX open, we have $\{\omega \varepsilon \Omega: x \in K(\omega), F(\omega, x) \cap U \neq \phi\} \varepsilon \Sigma$. Such an $F(.,$.$) is said to be u.s.c.$ (continuous, compact e.t.c.), if for all $\omega \varepsilon \Omega, F(\omega,$.$) is u.s.c. (continuous,$ compact e.t.c.) on $\mathrm{K}(\omega)$. Maps with stochastic domain were introduced by Eng1 [7]. A random fixed point of $F(.,$.$) is a measurable map x: \Omega+x$ s.t. for all $\omega \varepsilon \Omega, \quad x(\omega) \varepsilon K(\omega)$ and $x(\omega) \varepsilon F(\omega, x(\omega))$.

Finally, if $\mu()^{\prime}$ is a $\sigma$-finite measure on $\Sigma$ and $G: \Omega \rightarrow P_{f}(X)$ is measurable, we w11l denote by $S_{G}^{1}$ the set of integrable selectors of $G($.$) i.e.$ $S_{G}^{l}=\left\{g \varepsilon L^{1}(X): g(\omega) \varepsilon G(\omega) \mu-a . e.\right\}$. It is easy to check that this set is nonempty if and only if $\omega+\ln f\left\{\|x\|: x \in G(\omega)\right.$ belongs in $L_{+}^{1}$.

\section{RANDOM APPROXIMATIONS AND RANDOM FIXED POINTS.}

We will start with a random version of proposition 2.3 of Reich [6], which in turn was an extension of an earlier very interesting result of KyFan [5] (theorem 2).

In this section $(\Omega, \Sigma, \mu)$ is a complete $\sigma$-finite measure space. Also recall that a map $f: X \rightarrow X$ is nonexpansive, if $\|f(x)-f(y)\|<\|x-y\|$ for all $x, y \varepsilon X$. It is wel1 known (see for example Goebel-Reich [9]) that the metric projection on a closed, convex set in a Hilbert space, is nonexpansive. That's why in theorems $3.1,3.2,3.3$ 
and 3.4, that follow and involve the metric projection (either in their statement or in their proof), we assume that the ambient space is a Hilbert space.

THEOREM 3.1. If $X$ is a separable Hilbert space, $K: \Omega \rightarrow P_{f c}(X)$ is a separable multifunction and $f: G r K+X$ is a random, nonexpansive map, with stochastic domain $K($.$) s.t. for all \omega \varepsilon \Omega, f(\omega, K(\omega))$ is bounded. Then there exists $x: \Omega \rightarrow x$ measurable s.t. for a11 $\omega \varepsilon \Omega, x(\omega) \varepsilon K(\omega)$ and $\|x(\omega)-f(\omega)\|=d(f(\omega, x(\omega)), K(\omega))$.

PROOF. From theorem 3.4 of [13], we know that there exists $\hat{f}: \Omega x X+X \quad a$ Caratheodory extension of $f(.,$.$) (i.e. \omega+\hat{f}(\omega, x)$ is measurable, $x \rightarrow \hat{f}(\omega, x)$ is continuous and $\left.\left.\hat{\mathrm{f}}\right|_{G \mathrm{rK}}=\mathrm{f}\right)$. Let $\mathrm{p}(\omega): \mathrm{X}+\mathrm{K}(\omega)$ de the metric projection on $\mathrm{K}(\omega)$. We have already mentioned that $p(\omega)(\cdot)$ is nonexpansive and it is also easy to show (see [14]), that for every $z \varepsilon X, \omega \rightarrow p(\omega)(z)$ is measurable. Let $C(\omega)=\overline{\operatorname{conv}}(\operatorname{pof})(\omega, K(\omega))$. Note that $C(\omega)=\overline{\operatorname{conv}} \mathrm{y} \in D$ (pof) $(\omega, y)$, where $D$ is the countable set postulated from the separability of $K(\cdot)$. Hence $\omega \rightarrow C(\omega)$ is a measurable multifunction. For every $\omega \varepsilon \Omega,(p \circ f)(\omega,):. C(\omega)+C(\omega)$ and is nonexpansive. So from Browder [15], we know that it has a fixed point. Consider the multifunction $L: \Omega \rightarrow P_{f}(X)$ defined by:

$$
\begin{aligned}
L(\omega) & =\{x \in C(\omega):(\operatorname{pof})(\omega, x)=x\} \\
& =\{x \in C(\omega):(\operatorname{pof})(\omega, x)=x\} \\
G r L= & \{(\omega, x) \varepsilon \Omega x X:(\operatorname{pof})(\omega, x)=x\} \cap \operatorname{GrC}
\end{aligned}
$$

But $(\omega, x) \rightarrow(p o f)(\omega, x)$ is measurable in $\omega$ and continuous in $x$. Hence it is jointly measurable. Also since $C($.$) is measurable, \operatorname{GrC} \sum \sum_{x B}(X)$. Thus $\operatorname{GrL} \varepsilon \sum x B(X)$. Applying theorem 3 of Saint-Beuve [16], we get $x: \Omega \rightarrow x$ measurable s.t. $x(\omega) \varepsilon L(\omega)$ for all $\omega \varepsilon \Omega$. Therefore we have: $x(\omega) \varepsilon K(\omega)$ and $\|x(\omega)-f(\omega, x(\omega))\|=d(f(\omega, x(\omega)), K(\omega))$

REMARK 1. If $\mathrm{K}\left({ }_{\bullet}\right)$ is bounded values, the assumption of the range of $f(\omega,$.$) can$ be dropped.

REMARK 2. Another result in the direction of theorem 3.1 above with a different set of hypotheses, can be found in [17] (theorem 4).

We have a similar result for condensing maps. Recall that $f: X \rightarrow X$ is said to be $\gamma$-condensing, if it is continuous and for all Bc nonempty, bounded s.t. $\gamma(B)>0, \gamma(f(B))\left\langle\gamma(B)\right.$, where $\gamma\left({ }_{.}\right)$is the Kuratowski measure of noncompactness.

THEOREM 3.2. If $X$ is separable Hilbert space, $K: \Omega \rightarrow P_{f C}(X)$ is separable and $\mathrm{f}: \mathrm{GrK} \rightarrow \mathrm{X}$ is a random condensing map with stochastic domain $K($.$) s.t. for all$ $\omega \varepsilon \Omega, f(\omega, K(\omega))$ is bounded. Then there exists $x: \Omega+X$ measurable s.t. for all wES2 $x(\omega) \varepsilon K(\omega)$ and $\|x(\omega)-f(\omega, x(\omega))\|=d(f(\omega \cdot x(\omega)), K(\omega))$.

PROOF. Is the same as in theorem 3.1, using this time the fixed point of FuriVignoli [18].

Using theorem 3.1, we can have the following random version of a fixed point due to Browder-Pet ryshyn [4].

THEOREM 3.3. If $X$ is a separable Hilbert space, $K: \Omega \rightarrow P_{f c}(X)$ is separable with bounded values and $f: G r K \rightarrow X$ is a random, nonexpansive map with stochastic domain $K($.$) s.t. for every x \in \operatorname{bdK}(\omega)$ for which $p(\omega, f(\omega, x))=x$, we have $f(\omega, x)=x$. 
Then $f($. .) admits a random fixed point.

PROOF. From theorem 3.1 (see remark 1), we know that there exists $x: \Omega+X$ measurable s.t. $\|x(\omega)-f(\omega, x(\omega))\|=d(f(\omega, x(\omega)), K(\omega)))=\|f(\omega, x(\omega))-p(\omega, f(\omega, x))\|$. Since the best approximation is unique, $x(\omega)=p(\omega, f(\omega, x(\omega)))$. If $x(\omega) \in b d K(\omega)$, then by hypothesis $x(\omega)=f(\omega, x(\omega))$. Otherwise we must have that

$$
f(\omega, x(\omega)) \varepsilon K(\omega) \quad f(\omega, x(\omega))=p(\omega, f(\omega, x(\omega)))=x(\omega), \omega \varepsilon \Omega .
$$

REMARK. In the previous theorem, we can instead assume that for all $\omega \varepsilon \Omega f(\omega,$. is condensing on $K(\omega)$. Then in the proof we have to use theorem 3.2 .

Now we pass to multifunctions and prove the following random fixed point theorem.

THEOREM 3.4. If $X$ is a separable Hilbert space, $K: \Omega \rightarrow P_{f c}(X)$ is separable and $F: G r K \rightarrow P_{f c}(X)$ is an h-continuous, $\quad r$-condensing, random multifunction with stochastic domain $K($.$) s.t. for all \omega \varepsilon \Omega$ and for $x \in b d K(\omega), F(\omega, x) \cap p^{-1}(\omega, x)$

$\underline{c}\{x\}$ and $F(\omega, K(\omega))$ is bounded. Then $F(.,$.$) admits a random fixed point.$

PROOF. Let $G: \Omega_{X X} \rightarrow P_{f c}(X)$ be the multifunction defined by

$G(\omega, x)=F(\omega, p(\omega, x))$. From our hypotheses on $F(.,$.$) , we see that \omega \rightarrow G(\omega, x)$ is measurable, while $x \rightarrow G(\omega, x)$ is h-continuous. Also we claim that $G(\omega,$.$) is$

$\gamma$-condensing. So let $B c X$ be nonempty, bounded, with $Y(B)>0$. We have

$$
\gamma(G(\omega, B))=\gamma(F(\omega, p(\omega, B)))<\gamma(p(\omega, B))<\gamma(B)
$$

the last inequality being a consequence of the fact that $p(\omega,$.$) is nonexpansive.$ Let $\quad C(\omega)=\overline{\operatorname{convF}}(\omega, K(\omega))$. Then clearly $G(\omega,):. C(\omega) \rightarrow C(\omega)$. Note that if $\left\{x_{n}\right\}_{n \geq 1}$ is the countable set postulated from the separability of $K\left(f_{0}\right)$ and exploiting the h-continuity of $F\left(\omega_{0},\right)$, we have that $C(\omega)=\overline{\operatorname{conv}}_{n \geqslant 1} F\left(\omega, x_{n}\right) \quad \omega \rightarrow C(\omega) \quad$ is measurable. Then consider the multifunction defined by $L(\omega)=\{x \varepsilon C(\omega): x \in G(\omega, x)\}$. From theorem 1 of Himmelberg-Porter-Van Vleck [19], we know that for all $\omega \varepsilon \Omega, L(\omega) \neq 0$. Also note that $G r L=\{(\omega, x) \varepsilon \Omega x X: d(x, G(\omega, x))=0\} \cap \operatorname{GrC} \varepsilon \Sigma x B(X)$. Again theorem 3 of Saint-Beuve [16], produces a measurable map $x: \Omega+X$ s.t. $x(\omega) \varepsilon L(\omega)$, for all $\omega \varepsilon \Omega$. Let $\hat{\mathbf{x}}(\omega)=\mathrm{p}(\omega, \mathrm{x}(\omega))$. Clearly $\hat{\mathbf{x}}\left({ }_{\bullet}\right)$ is measurable and $\hat{\mathbf{x}}(\omega) \varepsilon \mathrm{bdK}(\omega)$. Then $x(\omega)=p^{-1}(\omega, \hat{x}(\omega))$ and $x(\omega) \varepsilon G(\omega, x(\omega))=F(\omega, \hat{x}(\omega)) \rightarrow x(\omega) \varepsilon p^{-1}(\omega, \hat{x}(\omega)) \cap$ $\mathrm{F}(\omega, \hat{x}(\omega)) \rightarrow \hat{x}(\omega)=x(\omega) \rightarrow x(\omega) \varepsilon K(\omega)$ and $x(\omega) \varepsilon F(\omega, x(\omega))$ i.e. $x($.$) is the desired$ random fixed point.

REMARK. If there is no $\omega$ dependence of the data in the previous theorem (deterministic case), then we can relax the hypotheses on $F\left(F_{-}\right.$) and simply assume that $F($.$) is closed, \gamma$-condensing and with bounded range. Also in the deterministic case, the theorem can be proved for general Banach spaces, if we assume that $K$ is approximatively w-compact and $F($.$) is w-u.s.c., with w-compact range. The proof is$ analogous to the random case and in the general Banach space, we have to use proposition 2.1 of Reich [6], which tells us that the metric projection on $K$ is $a$ wu.s.c. multifunction and eventually apply the Kakutani-KyFan fixed point theorem. Both those deterministic versions of theorem 3.4, extend theorem 3.3 of Reich [6]. Note that the second deterministic result that was stated in general Banach spaces, can not be extended to the random case, since as it was illustrated with a counter example in [20], a multifunction $G(\omega, x)$ which is measurable in $\omega$, wu.s.c. in $x$, is not in general jointly measurable. 
The next result extends theorem 8 of Engl [7]. Recall that a multifunction $F: K+2^{X} 、\{\phi\}$ is compact, if $\overline{F(K)}$ is compact. Also if $K_{c} X$ is convex $x \in \bar{K}$, we define $I(x, K)=\{z \varepsilon X: z=x+\lambda(y-x)$ for some $y \varepsilon X$ and $\lambda>0\}$. So $I(x, K)$ is nothing else, but the translation to the point $x$ of the well known from nonsmooth analysis, "Bouligand $t$ angent cone" to $K$ at $x$ (see Aubin-Ekeland [21]). Since we do not need any more the nonexpansiveness of the metric projection, the result can be stated for general separable Banach spaces.

THEOREM 3.5. If $X$ is separable Banach space, $K: \Omega \rightarrow P_{f c}(X)$ is separable and $\mathrm{F}: \mathrm{GrK} \rightarrow \mathrm{P}_{\mathrm{fc}}(\mathrm{X})$ is a compact, u.s.c., random multifunction with stochastic domain $K($.$) s.t. F(\omega, x) \subseteq I(x, K(\omega))$ wer. Then $F(.,$.$) a random fixed point.$

PROOF. From proposition 5 of Engl [7], we know that there exists a multifunction $H: G r K \rightarrow P_{f c}(X)$ s.t.

$$
\begin{aligned}
& \text { (i) for each }(\omega, x) \in G r K, H(\omega, x) \subseteq F(\omega, x) \\
& \text { (ii) for every } \omega \varepsilon \Omega, H(\omega .,) \text { is u.s.c. on } K(\omega) \\
& \text { (iii) } H(.,,) \text { is jointly measurable }
\end{aligned}
$$

Then let $L: \Omega \rightarrow 2^{X}$ be defined by

$$
L(\omega)=\{x \varepsilon K(\omega): x \in H(\omega, x)\}
$$

From theorem 3.1 of $\operatorname{Re} 1 \mathrm{ch}[6]$, we know that for al1 $\omega \varepsilon \Omega, L(\omega) \neq 0$. Observe that:

$$
\begin{aligned}
\operatorname{GrL} & =\left\{(\omega, x) \varepsilon \Omega_{X X} X: x \varepsilon K(\omega), x \varepsilon H(\omega, x)\right\} \\
& =\operatorname{proj}_{\Omega_{X X} X}((\Omega \times D) \cap G r H)
\end{aligned}
$$

where $D=\{(x, y) \varepsilon X x X: x=y\}$. But note that $\operatorname{GrH} \varepsilon \sum x B(X) x B(X)$. So

$(\Omega \times D) \cap \mathrm{GrH} \varepsilon \sum \mathrm{XB}(\mathrm{D})$. Then using the theorem in section 39.IV of Kuratowski [22], we get that $\operatorname{proj}_{\Omega_{X X}}(\Omega \times D) \cap \operatorname{GrF} \varepsilon \sum x B(X)$. Hence $\operatorname{GrL\varepsilon \Sigma xB}(X)$. Once again, through theorem 3 Saint-Beuve $[16]$, we get $x: \Omega \rightarrow X$ measurable s.t. $x(\omega) \varepsilon L(\omega), \omega \varepsilon \Omega \rightarrow x(\omega) \varepsilon H(\omega, x(\omega)) \underline{c F}(\omega, x(\omega))$.

\section{RANDOM DIFFERENTIAL INCLUSIONS •}

Let $(\Omega, \Sigma, \mu)$ be a complete probability space, $T=[0, b]$ a nonempty, closed, bounded interval in $R_{+}, X$ a finite dimensional Banach space and $x_{0}: \Omega \rightarrow X$ measurable. We consider the following random differential inclusion:

$$
\begin{aligned}
& x(\omega, t) \varepsilon F(\omega, t, x(\omega, t)) \text { a.e., for a } 11 \omega \in \Omega \\
& x(\omega, 0)=x_{0}(\omega)
\end{aligned}
$$

By random solution of $(*)$, we understand a stochastic process $x: \Omega x T \rightarrow X$, with absolutely continuous realizations, satisfying (*) a.e. in $t$, for all $w \varepsilon \Omega$.

In this section we present a theorem on the existence of random solutions of (*), 
that generalizes theorem 5.1 of [8].

THEOREM 4.1. If $F: \Omega \times T \times X \rightarrow P_{f c}(X)$ is a multifunction s.t.

(1) $(\omega, t, x) \rightarrow F(\omega, t, x)$ is measurable

(2) for all $(\omega, t) \varepsilon \Omega x T, x \rightarrow F(\omega, t, x)$ is u.s.c.

(3) $\quad|F(\omega, t, x)|<a(\omega, t)+b(\omega, t)\|x\| a . e .$, for a11 $\omega \varepsilon \Omega$, with $a(.,),. b(.,$.$) measurable$ and $a(\omega,),. b(\omega.) \varepsilon L_{+}^{1}$ Then $(*)$ admits a random solution.

PROOF. We will by determining an a priori bound for the random solutions of

(*) So let $x(.,$.$) be a random solution. Fixing \omega \varepsilon \Omega$, we have:

$$
\begin{aligned}
& x(\omega, t)=x_{0}(\omega)+\int_{0}^{t} f(s) d s, t \in T, f \in S_{F(\omega, x(\omega, .))}^{1} \\
& \|x(\omega, t)\|<\left\|x_{0}(\omega)\right\|+\int_{0}^{t}\|f(s)\| d s \\
& \|x(\omega t)\|<\left\|x_{0}(\omega)\right\|+\int_{0}^{t} a(\omega, s) d s+\int_{0}^{t} b(\omega, s)\|x(\omega, s)\| d s
\end{aligned}
$$

Applying Gronwall's inequality, we get that:

$$
\begin{aligned}
& \| x\left(\omega, t\left\|<\left(\left\|x_{0}(\omega)\right\|+\|a(\omega, .)\|_{1}\right) \exp \right\| b(\omega, .) \|_{1}=M(\omega)\right. \\
& \text { Set } \hat{F}(\omega, t, x)= \begin{cases}F(\omega, t, x) & \text { if }\|x\|<M(\omega) \\
F\left(\omega, t, \frac{M(\omega) x}{\|x\|}\right) \text { if }\|x\|>M(\omega)\end{cases}
\end{aligned}
$$

It is easy to check that $\hat{F}(., .,$.$) has the same measurability and semicontinuity$ properties as $F(., .,$.$) and furthermore we have that |\hat{F}(\omega, t, x)|<a(\omega, t)+b(\omega, t) M(\omega)$ $=\phi(\omega, t)$, with $\phi(.,$.$) measurable and \phi(\omega,.) \varepsilon \mathrm{L}_{+}^{+}$. We will consider (*) with the random orientor field $\hat{\mathrm{F}}(., .,$.$) .$

Let $W(\omega) \underline{c C}(T, X)$ be defined by:

$$
\begin{aligned}
& W(\omega)=\left\{x \in C(T, X): x(t)=x_{0}(\omega)+\int_{0}^{t} g(s) d s, t \varepsilon T,\|g(t)\|<\phi(\omega, t) \text { a.e. }\right\} \\
& \text { Define } \hat{T}: \Omega x C(T, X) x L^{1}(X)+C(T, X) \text { by: } \\
& \hat{T}(\omega, x, g)(t)=x_{0}(\omega)+\int_{0}^{t} g(s) d s-x(t)
\end{aligned}
$$

Clearly $\hat{T}(., .,$.$) is measurable in \omega$ and continuous in $(x, g)$. So is jointly measurable. Also if $\hat{B}(\omega)=B\left(0,\|\phi(\omega, .)\|_{1}\right)$ is the closed ball in $L^{1}(X)$, centered at the origin with radius $\|\phi(\omega, .)\|_{1}$, then $\hat{B}($.$) is measurable and$

$$
\mathrm{GrW}=\left\{(\omega, \mathrm{x}) \varepsilon \Omega \times \mathrm{CC}(\mathrm{T}, \mathrm{X}):\|\hat{\mathrm{T}}(\omega, \mathrm{x}, \mathrm{g})\|_{\infty}=0, \mathrm{~d}(\mathrm{~g}, \hat{\mathrm{B}}(\omega))=0\right\}
$$

$\mathrm{GRW} \varepsilon \Sigma_{\mathrm{XB}}(\mathrm{C}(\mathrm{T}, \mathrm{X}))$, , (since $\mathrm{g}=\mathrm{x}$, see Kurat owski [22], 39.IV). 
Furthermore, a simple application of the Arzela-Ascoli theorem, tells us that for every wES?, $W(\omega)$ is a compact subset of $C(T, X)$.

Next let $\hat{R}: G r W+2^{C(T, X) x L^{1}(X)}\{\phi\}$ be the multifunction defined by:

$$
\begin{gathered}
\hat{R}(\omega, x)=\left\{(y, f) \varepsilon C(T, X) x L^{1}(X): y(t)=x_{0}(\omega)+\int_{0}^{t} f(s) d s, t \varepsilon T, f \varepsilon S_{\hat{F}}^{1}(\omega, ., x(\cdot))\right. \\
\text { Define } \hat{G}: \Omega x C(T, X) x L^{1}(X) \rightarrow \mathbb{R}_{+} b y: \\
\hat{G}(\omega, x, f)=d\left(f, S_{\hat{F}(\omega, ., x(.))}^{1}\right)
\end{gathered}
$$

Note that:

$$
\begin{aligned}
& \mathrm{d}\left(\mathrm{f}, \mathrm{s}_{\hat{\mathrm{F}}(\omega, ., \mathrm{x}(.))}^{1}\right)=\inf \left\{\|\mathrm{f}-\mathrm{h}\|_{1} ; \mathrm{h} \varepsilon \mathrm{S}_{\hat{\mathrm{F}}(\omega, ., \mathrm{x}(.))}^{1}\right\} \\
& =\inf \left\{\int_{0}^{b}\|f(t)-h(t)\| d t: h \varepsilon S_{\hat{F}(\omega, ., x(.))}^{1}\right\} \\
& =\int_{0}^{b} \inf \{\|f(t)-z\|: z \varepsilon \hat{F}(w, t, x(t))\} d t \\
& =\int_{0}^{b} d(f(t), \hat{F}(\omega, t, x(t)) d t
\end{aligned}
$$

But by hypothesis (1), $(\omega, t, y)+d(z, \hat{\mathrm{F}}(\omega, t, y))$ a measurable and $z+d(z, \hat{F}(\omega, t, y))$ is continuous. Hence $(w, t, y, z) \rightarrow d(z, \hat{F}(\omega, t, y))$ is measurable. Also the evaluation map $(t, x(\cdot)) \rightarrow e_{t}(x(\cdot))=x(t)$, is continuous from $\operatorname{TxC}(T, X)$ into $X$. Hence we deduce that $(\omega, t, x(\cdot))+d(f(t), F(\omega, t, x(t)))$ is measurable from $\Omega_{\mathrm{XTXC}}(\mathrm{T}, \mathrm{X})$ into $\mathrm{IR}_{+}$. Rewrite $\hat{\mathrm{R}}(.,$.$) as follows:$

$$
\hat{R}(\omega, x)=\left\{(y, f) \varepsilon C(T, X) x L^{1}(x):\|\hat{T}(\omega, y, f)\|_{\infty}=0, \hat{G}(\omega, x, f)=0\right\}
$$

Let $P \underline{c C}(T, X) \times L^{1}(x)$ be defined by $P=\{(y, f): y=f\}$. Then the projection to the first variable is one-toone, continuous. Thus by Kuratowski [22] (39.IV):

$$
\begin{aligned}
& \operatorname{proj}_{\Omega x C(T, X) x C(T, X)} f \operatorname{rR}=\operatorname{proj}_{\Omega x C}(T, X) x C(T, x) \\
& ((G r W \cap P) \cap \\
& \left\{(\omega, x, y, f): y(0)=x_{0}(\omega), \hat{G}(\omega, x, f)=0\right\} \quad \varepsilon \Sigma x B(C(T, x)) x B(C(T, x)) .
\end{aligned}
$$

So if $R(\omega, x)=\operatorname{proj}_{C}(T, X) \hat{R}(\omega, x)$, then $\operatorname{GrR\varepsilon \Sigma } \times B(C(T, X)) \times B(C(T, X))$. Note that for fixed $\omega, R(\omega,):. W(\omega) \rightarrow W(\omega)$. We claim that it is u.s.c. To show this, since $W(\omega) \subseteq C(T, X)$ is compact, it suffices to show that $\operatorname{Gr}(\omega,$.$) is closed in$ $C(T, X) x C(T, X)$. So let $\left(x_{n}, y_{n}\right) \in G r R(\omega,),. n>1$ s.t. $\left(x_{n}, y_{n}\right)+(x, y)$. By definition:

$$
y_{n}(t)=x_{0}(\omega)+\int_{0}^{t} f_{n}(s) d s, t \varepsilon T, f_{n} \varepsilon S_{\hat{F}\left(\omega, \ldots, x_{n}(.)\right)}^{1}
$$

From the Dunford-Pettis compactness criterion, we deduce that $\overline{\left\{f_{n}\right\}}{ }_{n>1}^{w}$ is sequentially w-compact in $L^{1}(X)$. So by passing to a subsequence if necessary, we 


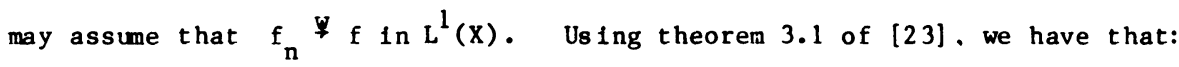

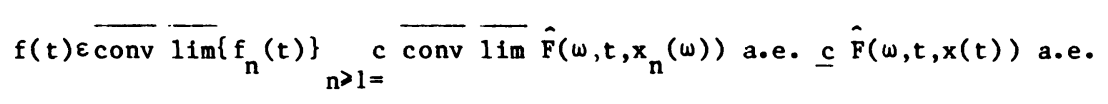

The last inclusion being a consequence of the upper semicontinuity and the

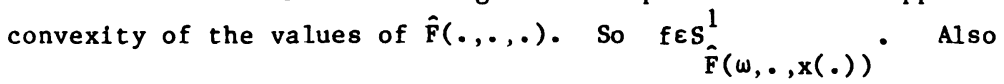

$$
\begin{aligned}
& y(t)=x_{0}(\omega)+\int_{0}^{t} f(s) d s, \quad t \in T \\
& (x, y) \varepsilon G r R(\omega . .) \\
& R(\omega .) \text { is u.s.c. from } W(\omega) \text { into } W(\omega) .
\end{aligned}
$$

Let $L: \Omega+2^{C(T, X)}$ be defined by:

$$
L(\omega)=\{x \varepsilon C(T, x): x \in R(\omega, x), x \in W(\omega)\}
$$

Since for fixed $\omega \varepsilon \Omega, R(\omega,):. W(\omega) \rightarrow W(\omega)$ is u.s.c., from the Kakutani-KyFan fixed point theorem, we have that $L(\omega) \neq \phi$, for every $\omega \varepsilon \Omega$. Then as in the proof of theorem 3.5, we have:

$$
G r L=\operatorname{proj}_{\Omega_{X} C(T, X)}\left(\left(\Omega_{x D}\right) \cap G r R\right) \varepsilon \Sigma \times B(C(T, X))
$$

where $D=\{(x, y) \varepsilon C(T, X) x C(T, X): x=y\}$. Apply theorem 3 of Saint-Beuve [16], to get $r: \Omega \rightarrow C(T, X)$ measurable, s.t. for all $\omega \varepsilon \Omega, r(\omega) \varepsilon L(\omega)$. Set $x(\omega, t)=r(\omega)(t)$. Clearly this is a random solution of $(*)$ with orientor field $F$. But from the definition of $F$, we see that $|F(\omega, t, x)|<a(\omega, t)+b(\omega, t)\|x\| a . e$, , for all $\omega \varepsilon \Omega$ and as in the beginning of the proof, through Gronwall's inequality, we get that

$\|x(\omega, t)\|<M(\omega) \rightarrow \hat{F}(\omega, t, x(\omega, t))=F(\omega, t, x(\omega, t)) \rightarrow x(\cdot,$.$) is the desired random$ solution of $(*)$.

ACKNOWLEDGEMENT. The author would like to express his gratitude to the referee for his helpful suggestions and remarks.

\section{REFERENCES}

1. HANS, 0. Random fixed point theorems, Transactions of the 1 st Prague Conf. on Information Theory, Statistics, Decision Functions and Random Processes, Czeschos1. Acad. Sc1., Prague (1957), pp. 105-125.

2. SPACEK, A. Zuffalige gleichungen, Czech. Math. Jour. $\underline{5}(80)$ (1955), pp. $462-466$.

3. BHARUCHA-REID, A. Random Integral Equations, Academic Press, New York (1972).

4. BROWDER, F. and PETRYSHYN, W. Construction of fixed points of nonlinear mappings in H1 lbert space, J. Math. Anal. Appl. 20 (1967), pp. 197-228.

5. K. Fan, Extensions of two fixed point theorems of F. Browder, Math. Z 112 (1969), pp. 234-240. 
6. REICH, S. Approximate selections, best approximations, fixed points and invariant sets, J. Math. Anal. App1. 62 (1978), pp. 104-113.

7. ENGL, H. Random fixed points for multivalued mappings, Pacific J. Math. 76 (1978), pp. 351-360.

8. PAPAGEORGIOU, N.S. Random differential inclusions in Banach spaces, J. Diff. Equations 65 (1986), pp. 287-303.

9. GOEBEL, K. and REICH, S. Uniform Convexity, Hyperbolic Geometry and Nonexpansive Mappings, Marce1 Dekker; New York and Base1 (1984.

10. AUBIN, J.P. and CELLINA, A. Differential Inclusions, Springer, Berlin (1983).

11. REICH, S. On fixed point theorems obtained from existence theorems for differential equations, J. Math. Anal. Appl. 54 (1976), pp. 26-36.

12. SCHAL, M. A selection theorem for optimization problems, Arch. Math. 25 (1974), pp. 219-223.

13. PAPAGEORGIOU, N.S. On measurable multifunctions with stochastic domain, J. Austr. Math. Soc. to appear.

14. KANDILAKIS, D. and PAPAGEORGIOU, N.S. Non-smooth analysis and approximation, J. Approx. Th. 51 (1987), pp. 104-125.

15. BROWDER, F. Fixed point theorems for noncompact mappings in Hilbert space, Proc. Nat. Acad. Sc1. U.S.A 53 (1965), pp. 1272-1276.

16. SAINT-BEUVE, M.F. On the extension of Von Neumann-Aumann's theorem, J. Funct. Ana 1. 17 (1974), pp. 112-129.

17. PAPAGEORGIOU, N.S. Random ixed point theorems for measurable multifunctions in Banach spaces, Proc. Amer. Math. Soc. 97 (1986), pp. 507-514.

18. FURI, M. and VIGNOLI, A. On $\alpha$-nonexpansive mappings and fixed points, Atti. Acad. Naz. Lincei. 48 (1970), pp. 195-198.

19. HIMMELBERG, C., PORTER, J., and VAN VLEK, F. Fixed point theorems for condensing multifunctions, Proc. Amer. Math. Soc. 23 (1969), pp. 635-641.

20. PAPAGEORGIOU, N.S. On measurable multifunctions with applications to random multivalued equations, Math. Japon. 32 (1987), pp. 437-464.

21. AUBIN, J.P. and EKELAND, I. Applied Nonlinear Analys1s, Wiley, New York (1984).

22. KURATOWSKI, K. Topology I, Academic Press, New York (1966).

23. PAPAGEORGIOU, N.S., Convergence theorems for Banach space valued integrable multifunctions, Intern. J. Math. and Math. Sc1. 10 (1987), pp. 433-442.

24. HIMMELBERG, C. Me asure relations, Fund. Math. 87 (1975), pp. 53-72.

25. WAGNER, D. Survey of measurable selection theorems, SIAM. J. Contr. Optim. 15 (1977), pp. 859-903. 


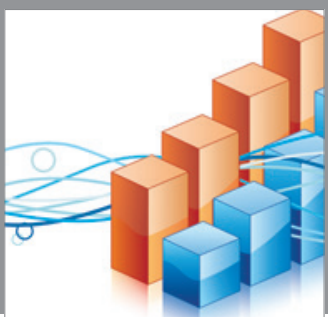

Advances in

Operations Research

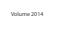

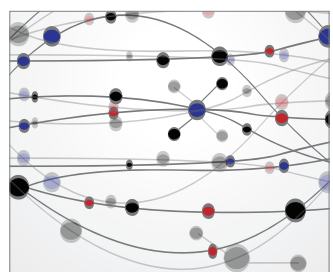

\section{The Scientific} World Journal
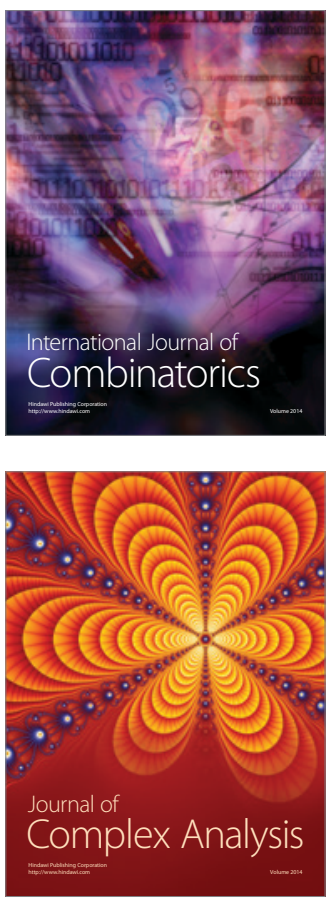

International Journal of

Mathematics and

Mathematical

Sciences
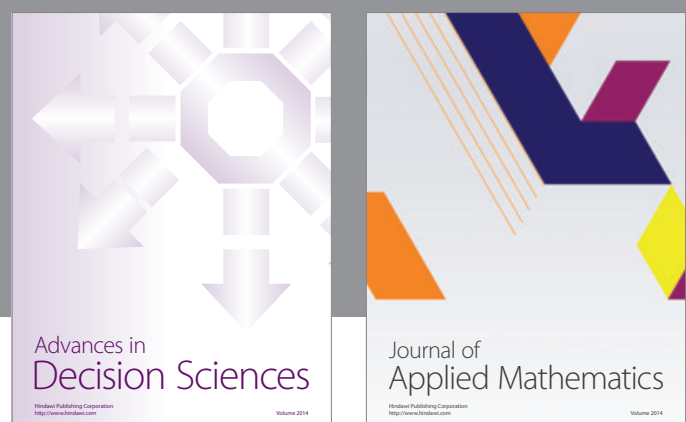

Journal of

Applied Mathematics
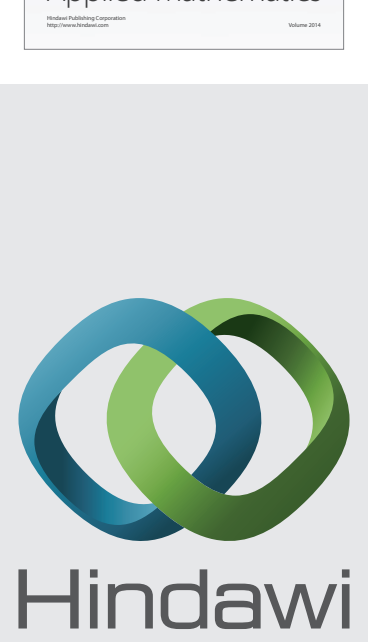

Submit your manuscripts at http://www.hindawi.com
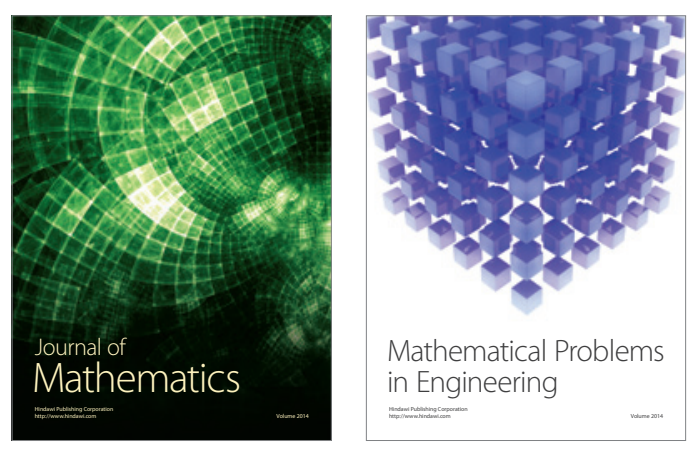

Mathematical Problems in Engineering
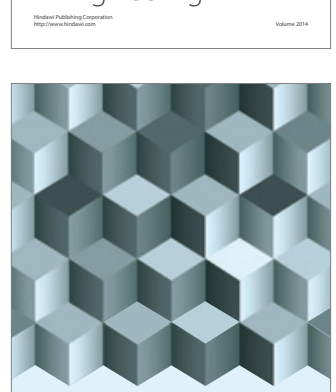

Journal of

Function Spaces
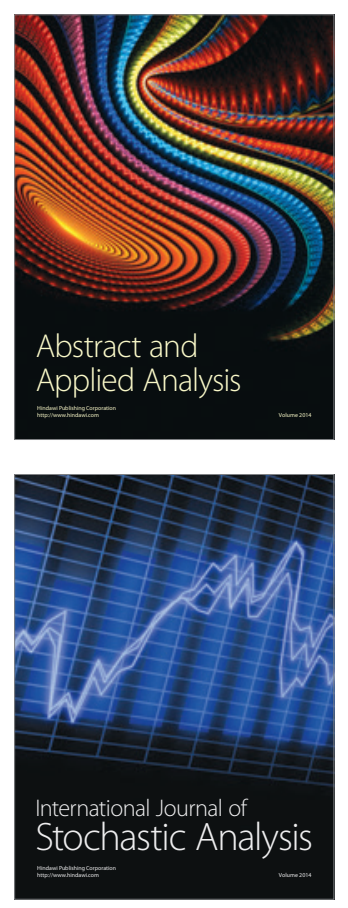

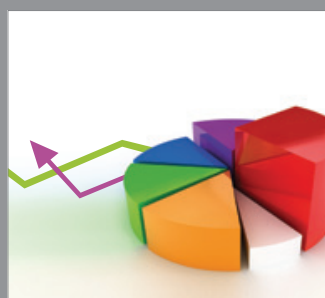

ournal of

Probability and Statistics

Promensencen
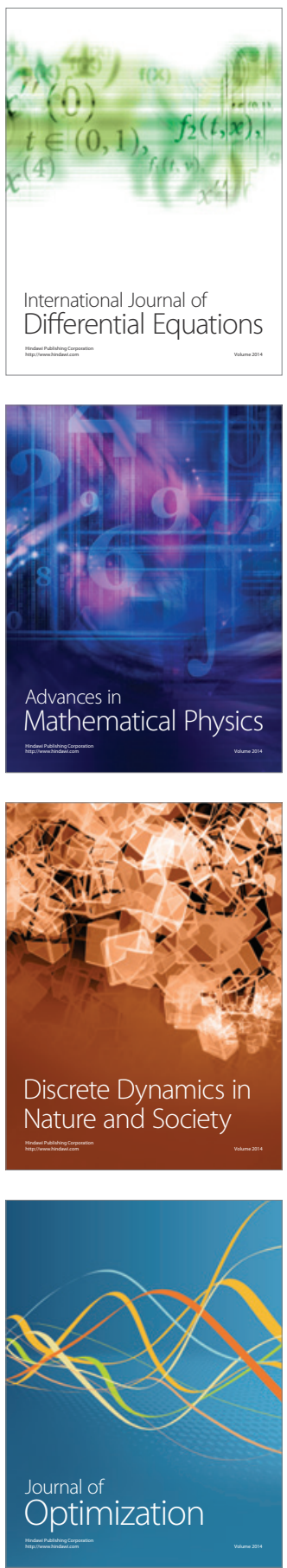